\title{
The continuous challenge of Chagas disease treatment: bridging evidence-based guidelines, access to healthcare, and human rights
}

\author{
Alberto Novaes Ramos Junior ${ }^{[1]}$ and Andréa Silvestre de Sousa ${ }^{[2],[3]}$ \\ [1]. Federal University of Ceara, School of Medicine, Department of Community Health, Fortaleza, Ceara, Brazil. \\ [2]. Oswaldo Cruz Foundation, Evandro Chagas National Institute of Infectious Diseases, Rio de Janeiro, Rio de Janeiro, Brazil. \\ [3]. Federal University of Rio de Janeiro, School of Medicine, Department of Internal Medicine, Rio de Janeiro, Rio de Janeiro, Brazil.
}

The high morbidity and mortality burden of Chagas disease is unquestionable, both in endemic and non-endemic settings ${ }^{1,2}$. The globalization of the disease is increasingly recognized and has thereby broadened the debate on the right to health, demanding an integrated response between countries ${ }^{1-3}$. Despite its relevance as a public health problem, great failures persist in national health systems in providing access to diagnosis and treatment for affected people ${ }^{3-6}$.

It is estimated that less than $90 \%$ of people who need specific treatment actually have access to $\mathrm{it}^{1,7}$. Primary health care not only plays a central role in the comprehensive care of people with Chagas disease as well as their families and communities; but also, in the surveillance and control of the disease ${ }^{1}$.

There are critical failings of science not only in developing new effective diagnostic methods, but also, new, safer and more effective therapeutic options ${ }^{1,6}$. In fact, the literature emphasizes the urgent need for the development of new treatments for chronic Chagas disease ${ }^{2,6}$. In this context, there are affected people with no guarantee of the right to health, who sustain the high burden of the disease and perpetuate the cycle of poverty in their families ${ }^{3,4}$. The primary care teams should be articulated with other levels of health care (greater technological complexity) for reference and counter-referencing of the more complex cases, keeping the clinical management also at the local level ${ }^{1,4}$.

The current political-economic scenario in Latin America has accelerated social inequalities, while at the same time weakening the role of the State in the development and regulation of health care actions, including surveillance and control of neglected diseases, such as Chagas disease ${ }^{1}$. In particular, Brazil in the last three years has regressed rapidly in

Corresponding author: Dr. Alberto Novaes Ramos Jr

e-mail: novaes@ufc.br

Received 19 December 2017

Accepted 22 December 2017 its social policies, with a deepening of income concentration, placing it again on the list of one of the most unequal countries in the world. This situation has enhanced individual and social vulnerability that was responsible for old and new determinants of disease transmission ${ }^{4}$. At the same time, the weakening of the health care network in the Unified Health System (SUS) further limits access to services, generating situations of serious program vulnerability, with a strong restriction on access to health, especially in areas of greater endemicity ${ }^{1}$.

Timely diagnosis and treatment are strategic to prevent disease progression and the occurrence of functional limitations, disability, and deficiency ${ }^{1}$. In addition to the etiological treatment, the comprehensive care of Chagas disease is very limited, especially, the chronic forms of the disease ${ }^{5,8}$. The chronic nature of the disease and the need to prevent disability and to rehabilitate the affected people create persisting challenges.

The disease-related stigma and the impact on quality of life enhances the cycle of neglect and poverty ${ }^{1,3}$. The different possible dimensions to care, such as psychological and social approaches, appear to be forgotten, further limiting access to health. Chagas disease should not be a definitive limiting factor to the social life and occupation of the affected persons. The follow-up of cases by the health team is fundamental for increased safety in the construction of quality of life.

Faced with the challenges presented, clinical guidelines should be developed systematically, based on the most recent scientific evidence, to support the decisions of health professionals. In addition, the best strategies for comprehensive care in the specific contexts of the countries must be provided for the people affected ${ }^{4,9}$. Besides the parasitological treatment, other aspects of care are fundamental to ensuring quality of life in people affected and must be evaluated. Hence, the functional capacity in Chagas disease, the effectiveness of non-surgical conservative treatment, and the cardiac involvement must be evaluated ${ }^{1}$.

However, given the complex and challenging scenarios required to achieve a balance; what are the real limitations 
between the availability of evidence-based guidelines, and the perceived and felt health needs amidst the different realities of the national health systems?

The cycle of neglect is partly accounted for by the weakness of the scientific evidences on the different perspectives of Chagas disease. Thus, the development of national guidelines for care, surveillance, and control of Chagas disease becomes a challenging task, dependent on the efforts of health professionals, researchers, and social movements 9 . The challenge of sustaining Chagas disease on the agenda of the national governments and the society has become a daily struggle ${ }^{1}$. Given this scenario, the professional capacity for clinical evaluation of Chagas disease has become a central reference for decision making.

The study titled Therapeutic drug monitoring of benznidazole and nifurtimox: A systematic review and quality assessment of published clinical practice guidelines by Oliveira MJ, et al. ${ }^{10}$ published in the Journal of the Brazilian Society of Tropical Medicine highlighted the importance of the need to critically analyze the national guidelines. The authors assessed the quality and consistency of recommendations on the pharmacological management of adults with chronic infection by Trypanosoma cruzi in the context of South America ${ }^{10}$. Five Clinical Practice Guidelines were selected from: Colombia, Argentina, Chile, Brazil, and Venezuela ${ }^{10}$.

Oliveira MJ, et al. suggested that there was no association between the methods used to develop the Clinical Practice Guidelines and the recommendations that were made ${ }^{10}$. The literature search in this study was restricted to articles published from January 2010 to March $2016^{10}$. In Brazil, a new Consensus on Chagas disease was published in December 2016; bringing in a more structured and evidence-based guideline ${ }^{1,4}$. Other countries, such as Bolivia, Colombia, and Argentina are in the process of developing new adjusted national guidelines for Chagas disease ${ }^{5,8}$.

In addition to the initial non-availability of evidence-based guidelines, there was also a lack of minimum standardization between and within the different countries, which created even greater obstacles for an integrated global response ${ }^{9,10}$. The authors emphasized that the assessment of the quality and consistency of the guidelines on the therapeutic drug monitoring of Chagas disease in adults with chronic phase resulted in documents of varying quality and also showed that none of the Clinical Practice Guidelines supported its evidence-based recommendations ${ }^{10}$. Significant differences were found on the pharmacological management, duration of treatment, standards of clinical controls, and the role of laboratory tests ${ }^{10}$. These differences can impact the access to health care contributing to worse health outcomes ${ }^{9,10}$.

Some national strategies have been developed in endemic countries as a solution to unmet demands in improving access to Chagas disease diagnosis and treatment ${ }^{1,9}$. In Bolivia, critical issues involve further scaling-up of diagnosis and treatment, and its sustainability in a scenario of limited local resources ${ }^{5}$. In Argentina, the development of an integrated strategy to decentralize diagnosis and treatment has been defined as a crucial issue in the national health systems. This must include a better inter-program coordination, enhancing existing monitoring and communication tools, and the mobilization of health care teams, especially in primary healthcare settings ${ }^{8}$.

The primary health care team must emphasize that, the main benefits of good clinical, labor, psychological, functional, and social management correspond especially to the etiological cure and prevention in the evolution of chronic heart disease and its consequences (social vulnerabilities, high cost of treatment, and death $)^{1,3,4}$.

In this analysis of Chagas disease guidelines, the domains of scope or purpose, stakeholder involvement, and clarity of presentation were better rated, while the domains of applicability and editorial independence received poor ratings ${ }^{10}$.

One of the objectives of the clinical treatment for T. cruzi infection must be to eliminate the parasites in the human hosts with specific drug treatment, to avoid the clinical syndrome that results from the irreversible lesions associated with the disease, and to give support to affected individuals and their families ${ }^{1,3,4}$. In addition, psychological and social effects must also be addressed ${ }^{1}$.

The morbidity and mortality burden of Chagas disease can be prevented or minimized by a combination of actions, at the level of individuals, families, communities, health and political systems $^{1}$. For the next 30 to 40 years, Chagas disease will still require the attention of governments, even if its transmission could be eliminated now, because of the burden of those with existing infections ${ }^{1,4}$.

The adoption of the Sustainable Development Goals - SDGs [Objetivos de Desenvolvimento Sustentável (ODS)], which included neglected tropical diseases (NTDs) as priority target for integrated global action, took place in September 2015 with endorsement by the 193 member-countries of the United Nations $(\mathrm{UN})^{11}$. This inclusion represented a unique opportunity to strengthen the agenda for the control of these diseases representing a significant public health problem throughout the world. Brazil as a signatory to the SDGs, has reinforced its historical responsibility, including its leadership in this process, by prioritizing health surveillance actions ${ }^{11}$.

In conclusion, clinical guidelines must be scientifically precise, with high responsibility to the people affected, the science and society. It must be predictable; providing specific details, defensible; showing high transparency about how they are developed and how consensus was reached, and applicable in a range of real world settings. Human and health rights should not be neglected when composing and reviewing clinical guidelines. The control of Chagas disease, as well as other NTDs, include a broader perspective of human and social development initiatives, with an inclusive perspective, thereby phasing out structural poverty in the endemic countries ${ }^{1}$.

Besides the necessity of linking the best available evidences and recommendations, we also emphasized the importance of ensuring the legitimate participation of representatives of people affected by the neglected diseases throughout the development process.

Expanding the social participation of people affected by Chagas disease is an important strategy for overcoming 
stigma, depression and fear of death, as well as strengthening empowerment, self-esteem, and self-care. The participation of the family in the plan of care for people affected by Chagas disease is fundamental.

\section{Conflict of interest}

The authors declare that there is no conflict of interest.

\section{REFERENCES}

1. Dias JCP, Ramos Jr AN, Gontijo ED, Luquetti A, Shikanai-Yasuda MA, Coura JR, et al. $2^{\text {nd }}$ Brazilian Consensus on Chagas Disease, 2015. Rev Soc Bras Med Trop. 2016;49(Suppl.I): 3-60.

2. Sperandio da Silva GM, Mediano MFF, Hasslocher-Moreno AM, Holanda MT, Silvestre de Sousa A, Sangenis LHC, et al. Benznidazole treatment safety: the Médecins Sans Frontières experience in a large cohort of Bolivian patients with Chagas' disease. J Antimicrob Chemother. 2017;72(9):2596-2601.

3. Pinheiro E, Brum-Soares L, Reis R, Cubides JC. Chagas disease: review of needs, neglect, and obstacles to treatment access in Latin America. Rev Soc Bras Med Trop. 2017;50(3):296-300.

4. Ramos Jr AN, Dias JC, Correia D. Towards control of Chagas disease: the contribution of the new Brazilian consensus. Rev Soc Bras Med Trop. 2016;49(Suppl 1):1-2.
5. Pinazo MJ, Pinto J, Ortiz L, Sánchez J, García W, Saravia R, et al. A strategy for scaling up access to comprehensive care in adults with Chagas disease in endemic countries: The Bolivian Chagas Platform. PLoS Negl Trop Dis. 2017;11(8):e0005770.

6. Sales Jr PA, Molina I, Fonseca Murta SM, Sánchez-Montalvá A, Salvador F, Corrêa-Oliveira R, et al. Experimental and Clinical Treatment of Chagas Disease: A Review. Am J Trop Med Hyg. 2017;97(5):1289-1303.

7. Chaves GC, Abi-Saab Arrieche M, Rode J, Mechali D, Reis PO, Alves RV, et al. Estimación de la demanda de medicamentos antichagásicos: una contribución para el acceso en América Latina. Rev Panam Salud Publica. 2017;41:e45. S1020-49892017000100502.

8. Klein K, Burrone MS, Alonso JP, Ares LR, Martí SG, Lavenia A, et al. Estrategia para mejorar el acceso al tratamiento etiológico para la enfermedad de Chagas en el primer nivel de atención en Argentina. Rev Panam Salud Publica. 2017;41:e20. S1020-49892017000100209.

9. Olivera MJ, Fory JA, Olivera AJ. Quality assessment of clinical practice guidelines for Chagas disease. Rev Soc Bras Med Trop. 2015;48(3):343-6.

10. Olivera MJ, Fory JA, Olivera AJ. Therapeutic drug monitoring of benznidazole and nifurtimox: A systematic review and quality assessment of published clinical practice guidelines. Rev Soc Bras Med Trop. 2017;50(6):748-755.

11. United Nations (UN). The Sustainable Development Goals Report 2017. Access 17 dec. 2017. Available from: https://unstats.un.org/ sdgs/. 